\title{
A one-pot synthesis of a 243-allyl dendrimer under ambient conditions
}

\author{
Cátia Ornelas, ${ }^{a}$ Jaime Ruiz Aranzaes, ${ }^{a}$ Eric Cloutet, ${ }^{b}$ Didier Astruc ${ }^{* a}$
}

\section{Supplementary Information Available}

\section{Characterizations of the dendrimers.}

\section{General}

Reagent-grade tetrahydrofuran (THF), diethyl ether were predried over $\mathrm{Na}$ foil and distilled from sodium-benzophenone anion under argon immediately prior to use. Methylene chloride $\left(\mathrm{CH}_{2} \mathrm{Cl}_{2}\right)$ was distilled from calcium hydride just before use. All other chemicals were used as received. All manipulations were carried out using Schlenk techniques or in a nitrogen-filled Vacuum Atmosphere drylab (glove-box).

${ }^{1} \mathrm{H}$ NMR spectra were recorded at $25^{\circ} \mathrm{C}$ with a Brucker AC $300(300 \mathrm{MHz})$ spectrometer. ${ }^{13} \mathrm{C}$ NMR spectra were obtained in the pulsed FT mode at $62.9 \mathrm{MHz}$ with a Brucker AC 300 spectrometer and ${ }^{29} \mathrm{Si}$ NMR spectra were obtained in at $59.6 \mathrm{MHz}$ with a Brucker AC 300 spectrometer. All chemical shifts are reported in parts per million $(\delta, \mathrm{ppm})$ with reference to $\mathrm{Me}_{4} \mathrm{Si}$ (TMS). Synthesis of the nonaallyl core 2 from 1 and of the phenol-triallyl dendron 4 were synthesized (Scheme 1, main text) as reported in references 5 and 7 of the main text.

Size Exclusion Chromatography (SEC): Size exclusions chromatography (SEC) of the dendrimers were performed using a JASCO HPLC pump type 880-PU, TOSOHAAS TSK gel columns, a Varian (séries RI-3) refractive index detector index detector and a JASCO $875 \mathrm{UV} / \mathrm{V}$ is absorption detector, with THF as the mobile phase.

\section{Nona-Cl dendrimer (3):}

${ }^{1} \mathrm{H} \mathrm{NMR}\left(\mathrm{CDCl}_{3}, 300 \mathrm{MHz}\right): 6.96$ (s, 3H, Ar), 1.65 (t, 18H, $\left.\mathrm{CH}_{2} \mathrm{CH}_{2} \mathrm{CH}_{2} \mathrm{Si}\right), 1.18$ (t, $18 \mathrm{H}, \mathrm{CH}_{2} \mathrm{CH}_{2} \mathrm{CH}_{2} \mathrm{Si}$ ), 0.77 (t, $18 \mathrm{H}, \mathrm{CH}_{2} \mathrm{CH}_{2} \mathrm{CH}_{2} \mathrm{Si}$ ), 0.36 (s, 54H, $\mathrm{SiMe}_{2}$ ). ${ }^{13} \mathrm{C} \mathrm{NMR}$ 
$\left(\mathrm{CDCl}_{3}, 62.9 \mathrm{MHz}\right.$ ): 148.9 (substituted arene $C$ ), 118.7 (unsubstituted arene $C$ ), 43.4 $\left(\mathrm{CH}_{2} \mathrm{CH}_{2} \mathrm{CH}_{2} \mathrm{Si}\right)$, 38.3 (benzylic quaternary $\left.\mathrm{C}\right), 17.8\left(\mathrm{CH}_{2} \mathrm{CH}_{2} \mathrm{CH}_{2} \mathrm{Si}\right.$, and $\left.\mathrm{CH}_{2} \mathrm{CH}_{2} \mathrm{CH}_{2} \mathrm{Si}\right),-3.2\left(\mathrm{Si}\left(\mathrm{CH}_{3}\right)_{2} \mathrm{Cl}\right) .{ }^{29} \mathrm{Si} \mathrm{NMR}\left(\mathrm{CDCl}_{3}, 59.6 \mathrm{MHz}\right): 32.7$ ( $\left.\mathrm{Si}-\mathrm{Cl}\right)$.

\section{7-olefin dendrimer (5):}

${ }^{1} \mathrm{H} \mathrm{NMR}\left(\mathrm{CDCl}_{3}, 300 \mathrm{MHz}\right): 7.22$ and 6.76 (double d, 36H, p. $\left.\mathrm{C}_{6} H_{4}\right), 7.04$ (s, 3H, arene core), $5.52\left(\mathrm{~m}, 27 \mathrm{H}, \mathrm{CH}=\mathrm{CH}_{2}\right), 4.96\left(\mathrm{t}, 54 \mathrm{H}, \mathrm{CH}=\mathrm{CH}_{2}\right), 2.40\left(\mathrm{~d}, 54 \mathrm{H}, \mathrm{CH}_{2}-\mathrm{CH}=\mathrm{CH}_{2}\right)$, $1.68\left(\mathrm{t}, 18 \mathrm{H}, \mathrm{CH}_{2} \mathrm{CH}_{2} \mathrm{CH}_{2} \mathrm{Si}\right), 1.20\left(\mathrm{t}, 18 \mathrm{H}, \mathrm{CH}_{2} \mathrm{CH}_{2} \mathrm{CH}_{2} \mathrm{Si}\right), 0.70$ (t, $18 \mathrm{H}$, $\left.\mathrm{CH}_{2} \mathrm{CH}_{2} \mathrm{CH}_{2} \mathrm{Si}\right), 0.16$ (s, 54H, $\left.\mathrm{SiMe}_{2}\right) .{ }^{13} \mathrm{C} \mathrm{NMR}\left(\mathrm{CDCl}_{3}, 62.9 \mathrm{MHz}\right): 154.1\left(\mathrm{OC}_{\mathrm{Ar}}\right)$, 139.6 (substituted arene core $C$ ), 135.8 (inner $C=\mathrm{C}$ ), 128.7 and 120.5 (unsubstituted arene $C$ of the dendron), 118.6 (outer $\mathrm{C}=C$ ), $43.8\left(\mathrm{CH}_{2}-\mathrm{CH}=\mathrm{CH}_{2}\right.$ and $\mathrm{CH}_{2} \mathrm{CH}_{2} \mathrm{CH}_{2} \mathrm{Si}$ ), 43.1 (benzylic quaternary $C$ of the core and dendron), $17.9\left(\mathrm{CH}_{2} \mathrm{CH}_{2} \mathrm{CH}_{2} \mathrm{Si}\right), 16.6$ $\left(\mathrm{CH}_{2} \mathrm{CH}_{2} \mathrm{CH}_{2} \mathrm{Si}\right),-0.0036\left(\mathrm{Si}\left(\mathrm{CH}_{3}\right)_{2} \mathrm{O}\right) .{ }^{29} \mathrm{Si} \mathrm{NMR}\left(\mathrm{CDCl}_{3}, 59.6 \mathrm{MHz}\right): 18.78(\mathrm{Si}-\mathrm{O})$. MALDI-TOF: Calc. for $\mathrm{C}_{198} \mathrm{H}_{282} \mathrm{Si}_{9} \mathrm{O}_{9}: 3059.12$; found: $3079.06\left(\mathrm{MNa}^{+}\right)$. The spectrum (vide infra) shows several peaks in addition to the molecular peak. We suggest that these peaks result from fragmentation rather than from uncomplete reaction because (i) excess silane was used and its reaction is easy and fast (which is confirmed by NMR), (ii) fragmentation is found for both cleavages before and beyond the oxygen atom (see the structures of the cleaved fragments on the spectrum).

\section{7-Cl dendrimer (6):}

${ }^{1} \mathrm{H} \mathrm{NMR}\left(\mathrm{CDCl}_{3}, 300 \mathrm{MHz}\right): 7.12$ and 6.74 (double d, 108H, p. $\left.\mathrm{C}_{6} H_{4}\right), 7.03$ (s, 3H, arene core), 1.65 (t, 54H, $\left.\mathrm{CH}_{2} \mathrm{CH}_{2} \mathrm{CH}_{2} \mathrm{Si}\right), 1.17$ (t, 54H, $\left.\mathrm{CH}_{2} \mathrm{CH}_{2} \mathrm{CH}_{2} \mathrm{Si}\right), 0.75$ (t, 54H, $\left.\mathrm{CH}_{2} \mathrm{CH}_{2} \mathrm{CH}_{2} \mathrm{Si}\right), 0.34$ (s, 162H, $\left.\mathrm{SiMe}_{2}\right) .{ }^{13} \mathrm{C} \mathrm{NMR}\left(\mathrm{CDCl}_{3}, 62.9 \mathrm{MHz}\right): 151.9\left(\mathrm{OC}_{\mathrm{Ar}}\right)$, 137.1 (benzylic substituted $C_{\mathrm{Ar}}$ ), 124.4 and 117.1 (unsubstituted arene $C$ of the dendron), $40.4\left(\mathrm{CH}_{2} \mathrm{CH}_{2} \mathrm{CH}_{2} \mathrm{Si}\right), 38.3$ (benzylic quaternary $\mathrm{C}$ of the dendron), 18.0 $\left(\mathrm{CH}_{2} \mathrm{CH}_{2} \mathrm{CH}_{2} \mathrm{Si}\right), 16.3\left(\mathrm{CH}_{2} \mathrm{CH}_{2} \mathrm{CH}_{2} \mathrm{Si}\right),-0.0079\left(\mathrm{Si}\left(\mathrm{CH}_{3}\right)_{2} \mathrm{O}\right),-3.2\left(\mathrm{Si}\left(\mathrm{CH}_{3}\right)_{2} \mathrm{Cl}\right) .{ }^{29} \mathrm{Si}$ NMR $\left(\mathrm{CDCl}_{3}, 59.6 \mathrm{MHz}\right): 31.66(\mathrm{Si}-\mathrm{Cl}), 18.99(\mathrm{Si}-\mathrm{O})$. 
81-olefin dendrimer (7):

${ }^{1} \mathrm{H}$ NMR $\left(\mathrm{CDCl}_{3}, 300 \mathrm{MHz}\right): 7.11$ and 6.73 (double d, 324H, p. $\left.\mathrm{C}_{6} H_{4}\right), 5.54(\mathrm{~m}, 81 \mathrm{H}$, $\left.\mathrm{CH}=\mathrm{CH}_{2}\right), 4.96\left(\mathrm{t}, 162 \mathrm{H}, \mathrm{CH}=\mathrm{CH}_{2}\right), 2.40\left(\mathrm{~d}, 162 \mathrm{H}, \mathrm{CH}_{2}-\mathrm{CH}=\mathrm{CH}_{2}\right), 1.62(\mathrm{t}, 72 \mathrm{H}$, $\mathrm{CH}_{2} \mathrm{CH}_{2} \mathrm{CH}_{2} \mathrm{Si}$ ), 1.17 (t, 72H, $\mathrm{CH}_{2} \mathrm{CH}_{2} \mathrm{CH}_{2} \mathrm{Si}$ ), 0.66 (t, 72H, $\mathrm{CH}_{2} \mathrm{CH}_{2} \mathrm{CH}_{2} \mathrm{Si}$ ), 0.15 (s, 216H, $\left.\mathrm{SiMe}_{2}\right) \cdot{ }^{13} \mathrm{C} \mathrm{NMR}\left(\mathrm{CDCl}_{3}, 62.9 \mathrm{MHz}\right): 154.3\left(\mathrm{OC}_{\mathrm{Ar}}\right), 139.1$ (benzylic substituted $C_{\mathrm{Ar}}$ ), 135.6 (inner $C=\mathrm{C}$ ), 128.5 and 120.4 (unsubstituted arene $C$ of the dendron), 118.7 (outer $\mathrm{C}=\mathrm{C}), 43.8\left(\mathrm{CH}_{2}-\mathrm{CH}=\mathrm{CH}_{2}\right.$ and $\left.\mathrm{CH}_{2} \mathrm{CH}_{2} \mathrm{CH}_{2} \mathrm{Si}\right), 43.1$ (benzylic quaternary $C$ of the dendron), $17.9\left(\mathrm{CH}_{2} \mathrm{CH}_{2} \mathrm{CH}_{2} \mathrm{Si}\right), 16.6\left(\mathrm{CH}_{2} \mathrm{CH}_{2} \mathrm{CH}_{2} \mathrm{Si}\right),-0.0036\left(\mathrm{Si}\left(\mathrm{CH}_{3}\right)_{2} \mathrm{O}\right) .{ }^{29} \mathrm{Si}$ NMR $\left(\mathrm{CDCl}_{3}, 59.6 \mathrm{MHz}\right): 18.76(\mathrm{Si}-\mathrm{O})$.

\section{1-Cl dendrimer $(\mathbf{8})$ :}

${ }^{1} \mathrm{H}$ NMR $\left(\mathrm{CDCl}_{3}, 300 \mathrm{MHz}\right): 7.10$ and 6.72 (double d, 324H, p. $\left.\mathrm{C}_{6} H_{4}\right), 1.62(\mathrm{t}, 234 \mathrm{H}$, $\left.\mathrm{CH}_{2} \mathrm{CH}_{2} \mathrm{CH}_{2} \mathrm{Si}\right), 1.15\left(\mathrm{t}, 234 \mathrm{H}, \mathrm{CH}_{2} \mathrm{CH}_{2} \mathrm{CH}_{2} \mathrm{Si}\right), 0.75\left(\mathrm{t}, 234 \mathrm{H}, \mathrm{CH}_{2} \mathrm{CH}_{2} \mathrm{CH}_{2} \mathrm{Si}\right), 0.34$ (s, 702H, $\left.\mathrm{SiMe}_{2}\right) .{ }^{13} \mathrm{C} \mathrm{NMR}\left(\mathrm{CDCl}_{3}, 62.9 \mathrm{MHz}\right): 150.9\left(\mathrm{OC}_{\mathrm{Ar}}\right), 138.1$ (benzylic substituted $\left.C_{\mathrm{Ar}}\right), 125.4$ and 117.7 (unsubstituted arene $C$ of the dendron), $41.4\left(\mathrm{CH}_{2} \mathrm{CH}_{2} \mathrm{CH}_{2} \mathrm{Si}\right)$, 39.3 (benzylic quaternary $C$ of the dendron), $17.8 \quad\left(\mathrm{CH}_{2} \mathrm{CH}_{2} \mathrm{CH}_{2} \mathrm{Si}\right), 15.3$ $\left(\mathrm{CH}_{2} \mathrm{CH}_{2} \mathrm{CH}_{2} \mathrm{Si}\right),-0.0087\left(\mathrm{Si}\left(\mathrm{CH}_{3}\right)_{2} \mathrm{Cl}\right),-3.1\left(\mathrm{Si}\left(\mathrm{CH}_{3}\right)_{2} \mathrm{O}\right) .{ }^{29} \mathrm{Si}$ NMR $\left(\mathrm{CDCl}_{3}, 59.6\right.$ MHz): 31.6 (Si-Cl), 19.2 (outer Si-O), 18.8 (inner Si-O).

234-olefin dendrimer (9):

${ }^{1} \mathrm{H}$ NMR $\left(\mathrm{CDCl}_{3}, 300 \mathrm{MHz}\right.$ ): 7.10 and 6.72 (double d, 468H, p. $\left.\mathrm{C}_{6} H_{4}\right), 5.53(\mathrm{~m}, 243 \mathrm{H}$, $\left.\mathrm{CH}=\mathrm{CH}_{2}\right), 4.95\left(\mathrm{t}, 486 \mathrm{H}, \mathrm{CH}=\mathrm{CH}_{2}\right), 2.39\left(\mathrm{~d}, 486 \mathrm{H}, \mathrm{CH}_{2}-\mathrm{CH}=\mathrm{CH}_{2}\right), 1.63(\mathrm{t}, 234 \mathrm{H}$, $\mathrm{CH}_{2} \mathrm{CH}_{2} \mathrm{CH}_{2} \mathrm{Si}$ ), 1.16 (t, 234H, $\left.\mathrm{CH}_{2} \mathrm{CH}_{2} \mathrm{CH}_{2} \mathrm{Si}\right), 0.66$ (t, 234H, $\mathrm{CH}_{2} \mathrm{CH}_{2} \mathrm{CH}_{2} \mathrm{Si}$ ), 0.14 (s, 702H, $\left.\mathrm{SiMe}_{2}\right) .{ }^{13} \mathrm{C} \mathrm{NMR}\left(\mathrm{CDCl}_{3}, 62.9 \mathrm{MHz}\right): 153.3\left(\mathrm{OC}_{\mathrm{Ar}}\right), 138.7$ (benzylic substituted $C_{\mathrm{Ar}}$ ), 135.0 (inner $C=\mathrm{C}$ ), 128.0 and 119.8 (unsubstituted arene $C$ of the dendron), 117.9 
(outer $\mathrm{C}=\mathrm{C}$ ), $43.5\left(\mathrm{CH}_{2}-\mathrm{CH}=\mathrm{CH}_{2}\right), 43.1\left(\mathrm{CH}_{2} \mathrm{CH}_{2} \mathrm{CH}_{2} \mathrm{Si}\right), 42.3$ (benzylic quaternary $\mathrm{C}$ of the dendron), $17.8\left(\mathrm{CH}_{2} \mathrm{CH}_{2} \mathrm{CH}_{2} \mathrm{Si}\right), 17.4\left(\mathrm{CH}_{2} \mathrm{CH}_{2} \mathrm{CH}_{2} \mathrm{Si}\right),-0.85\left(\mathrm{Si}\left(\mathrm{CH}_{3}\right)_{2} \mathrm{O}\right) .{ }^{29} \mathrm{Si}$ NMR $\left(\mathrm{CDCl}_{3}, 59.6 \mathrm{MHz}\right)$ : 18.88 (outer Si-O), 18.90 (inner Si-O) . 


\section{${ }^{1} \mathrm{H}$ NMR of 2}

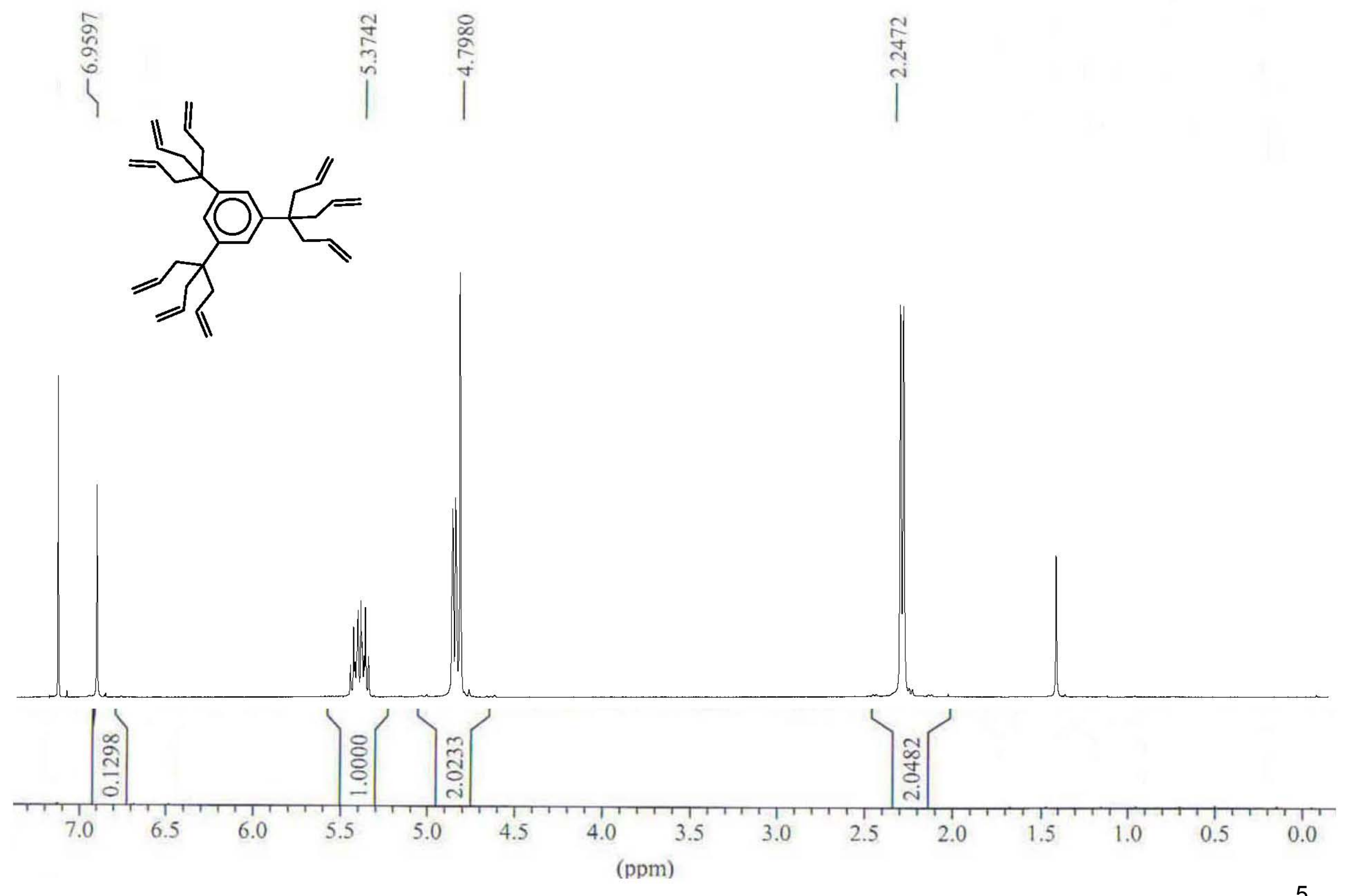




\section{${ }^{1} \mathrm{H}$ NMR of 3}
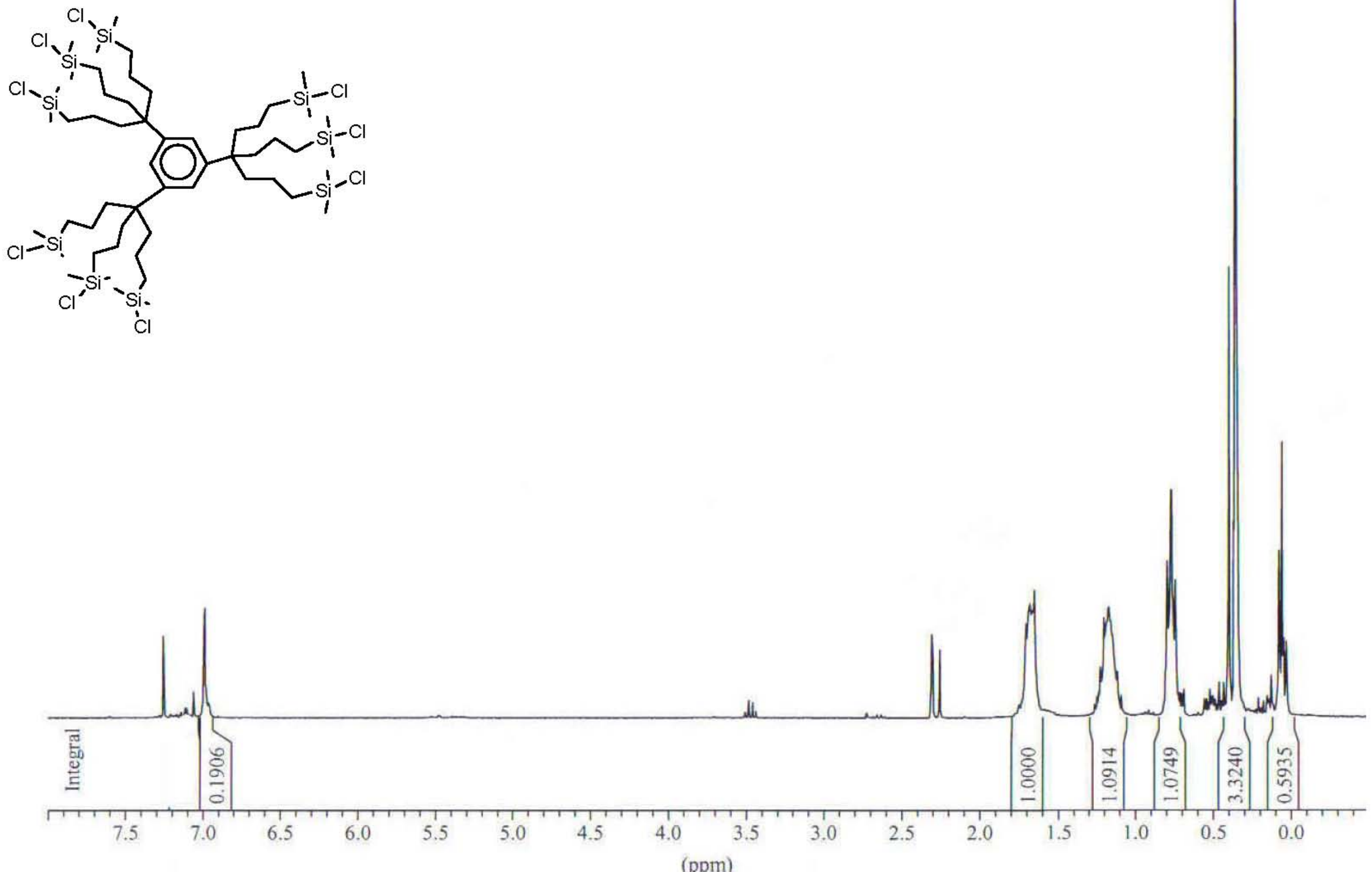


\section{${ }^{1} \mathrm{H}$ NMR of 5}

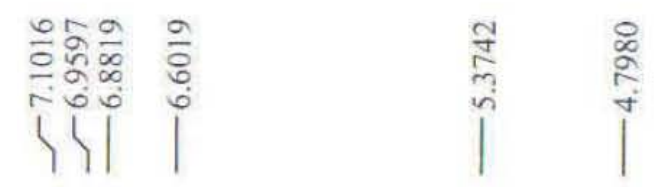
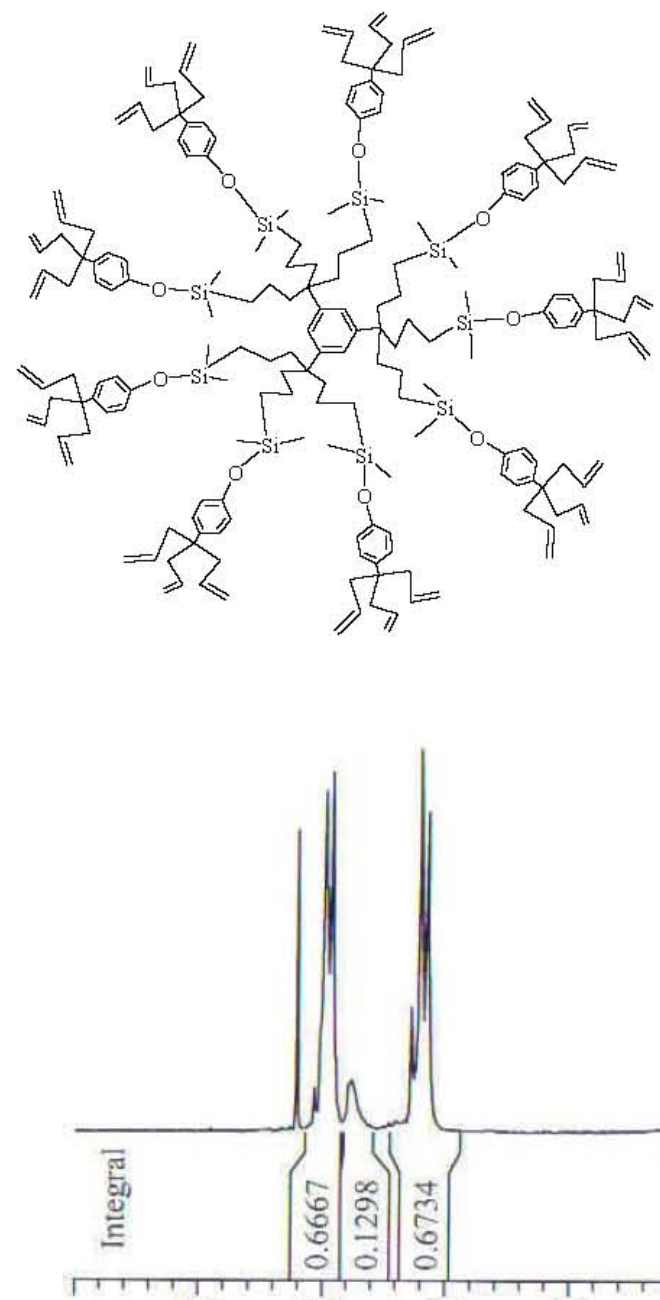


\section{${ }^{1} \mathrm{H}$ NMR of 6}

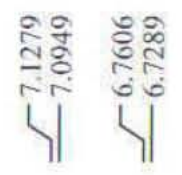

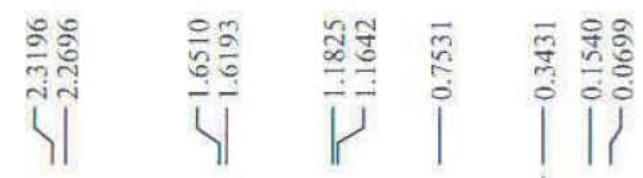
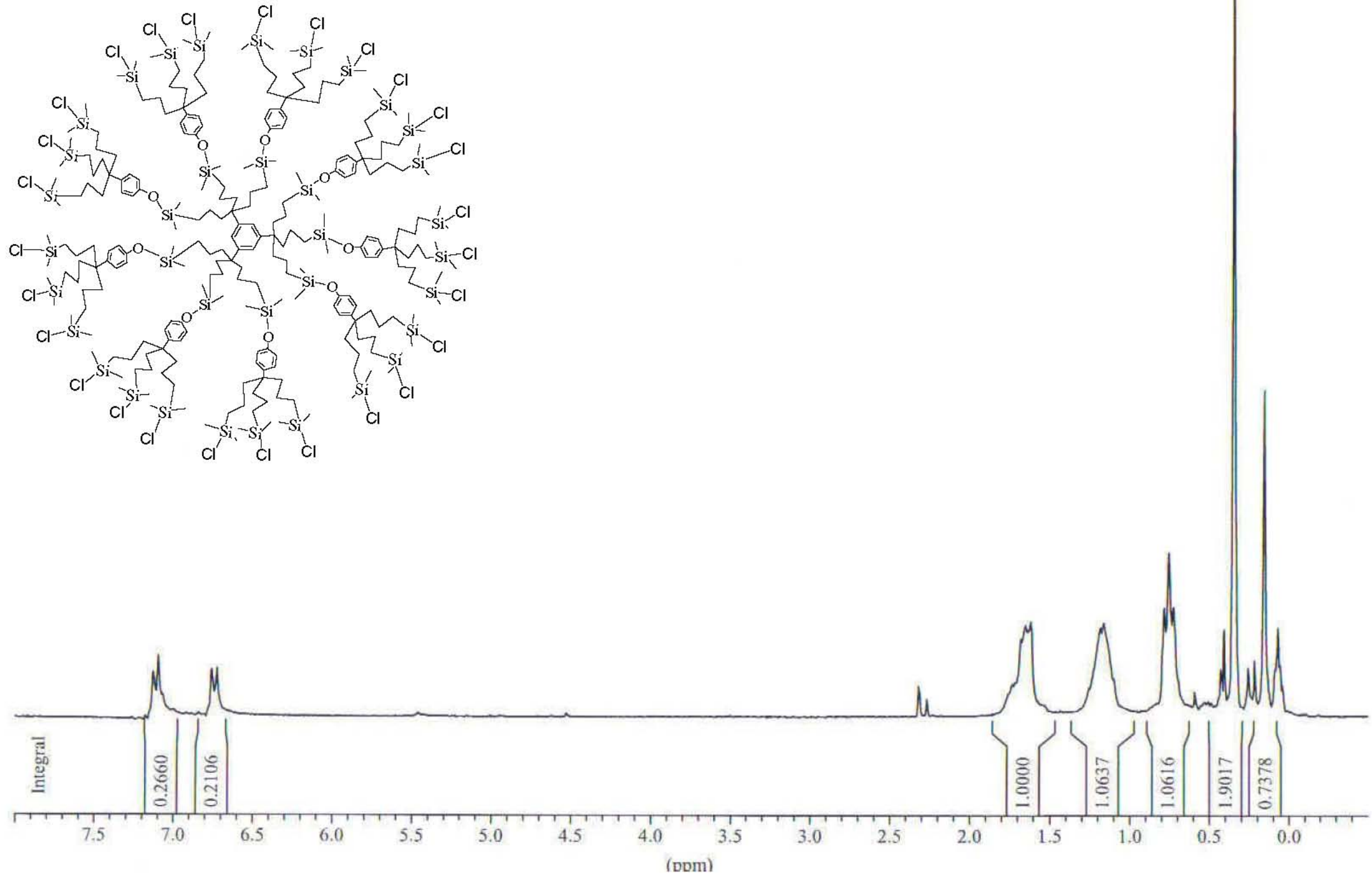


\section{${ }^{1} \mathrm{H}$ NMR of 7}

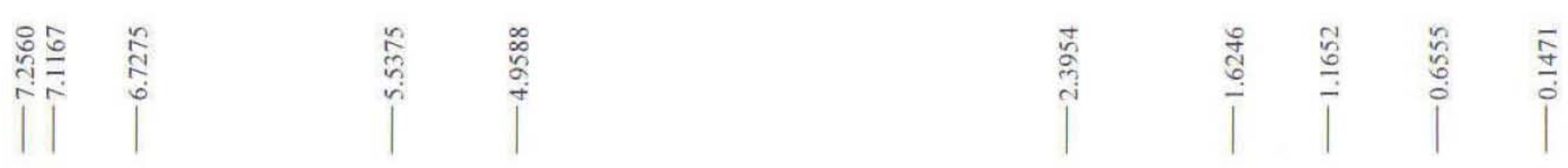
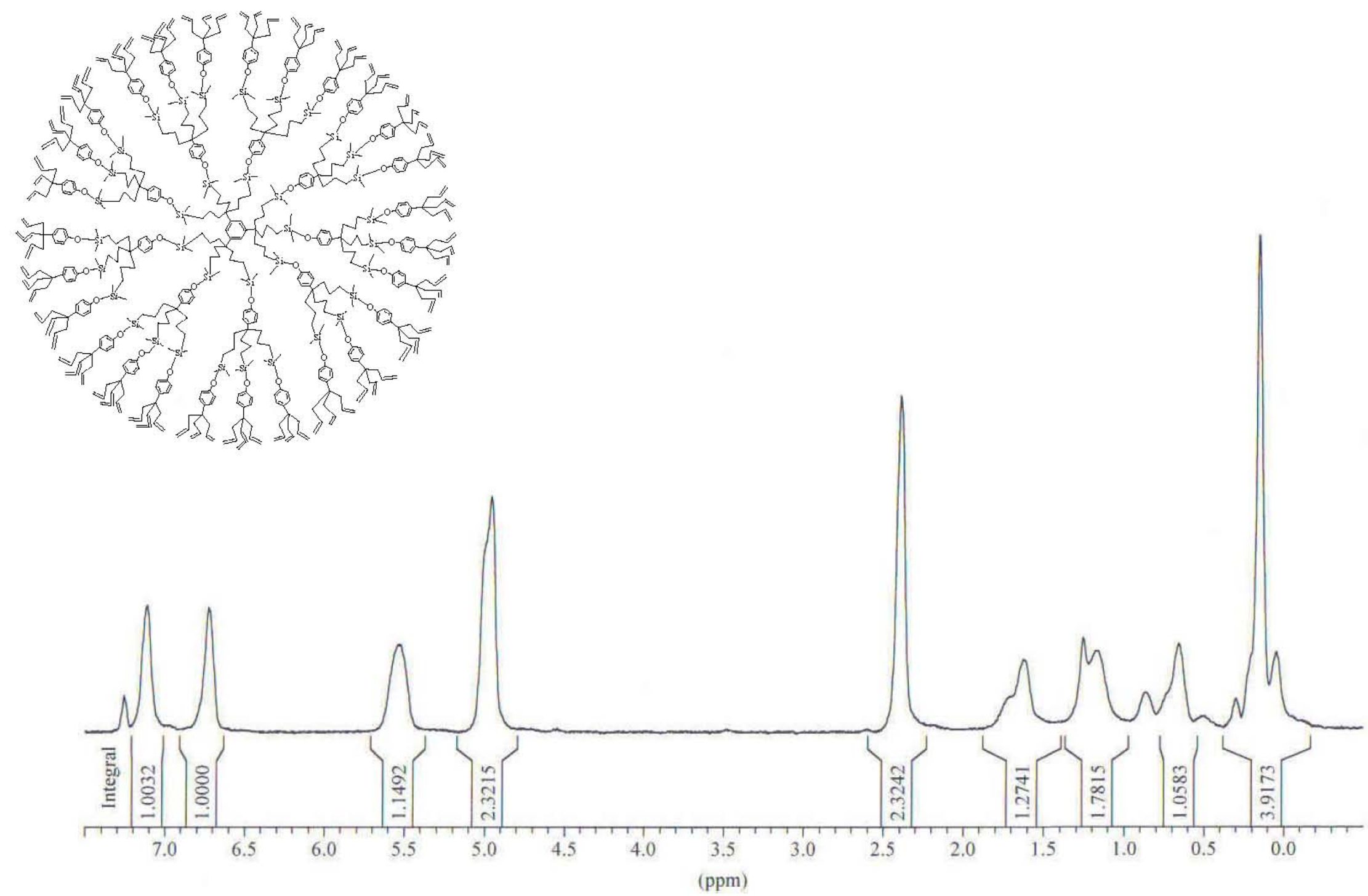


\section{${ }^{1} \mathrm{H}$ NMR of 8}

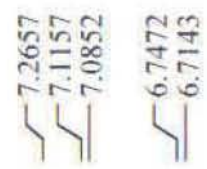

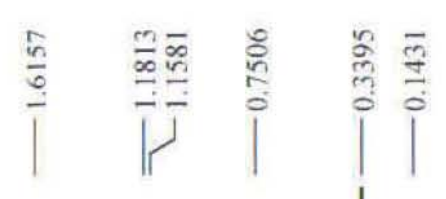
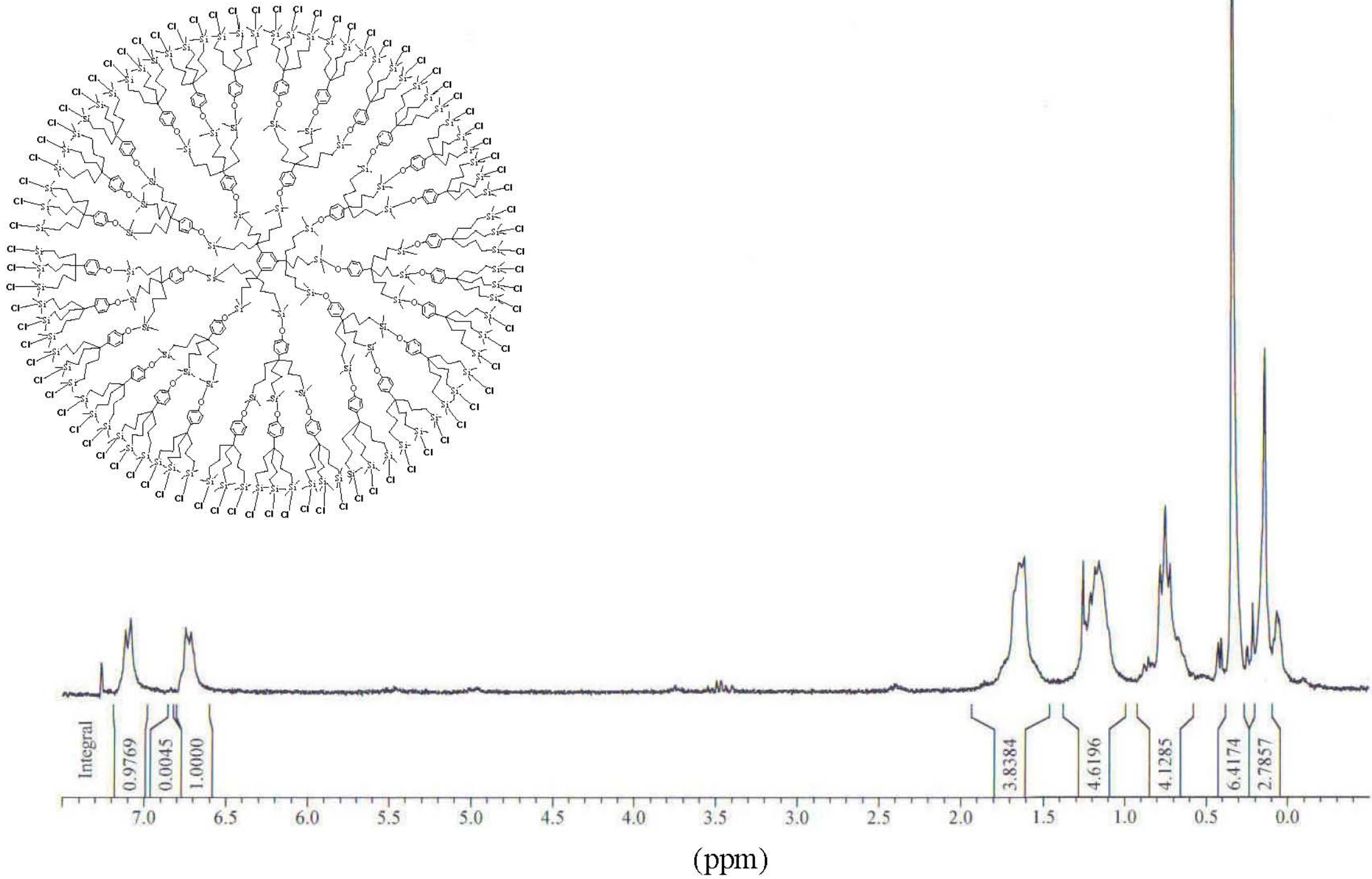


\section{${ }^{1} \mathrm{H}$ NMR of 9}

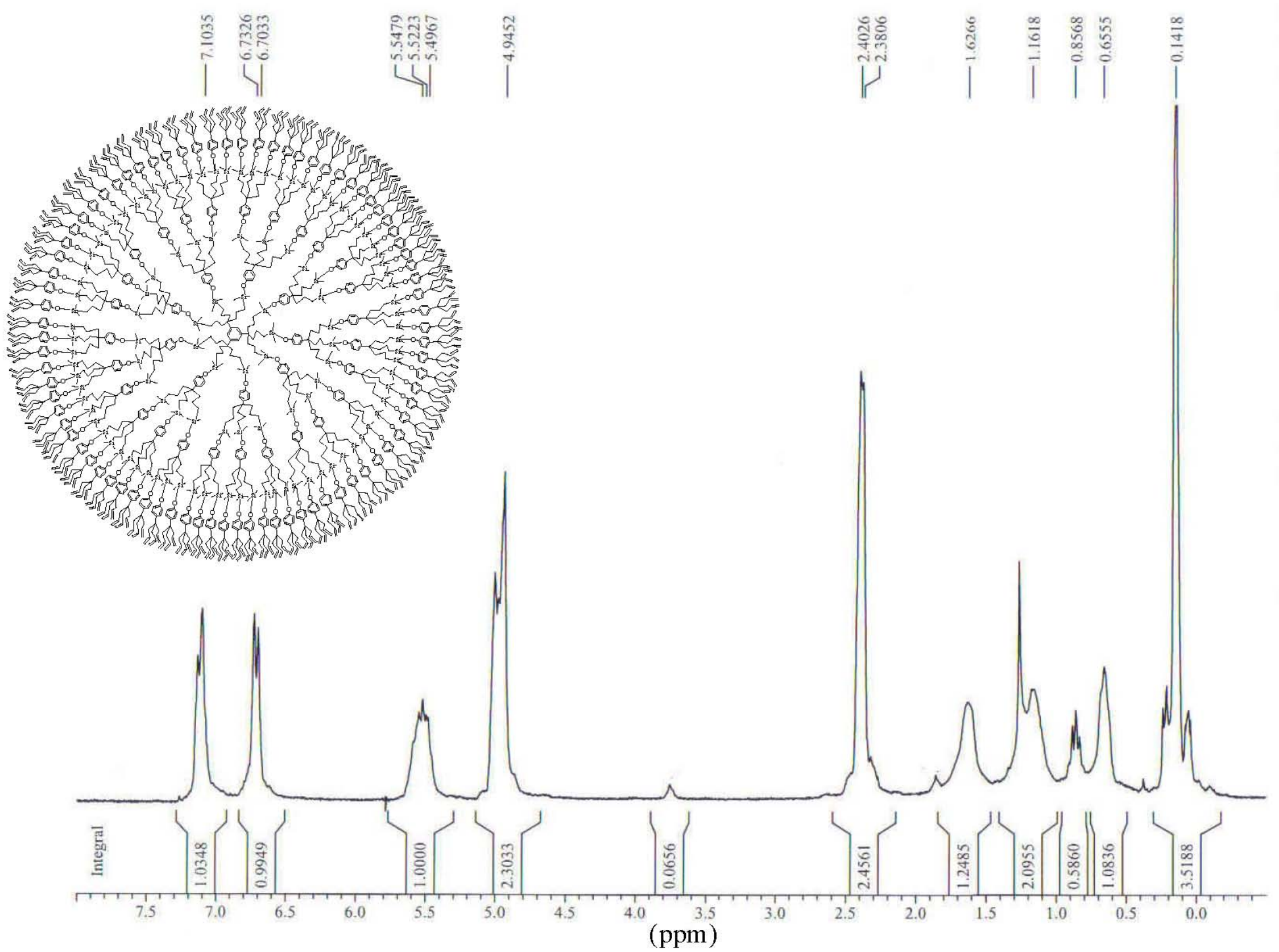


${ }^{29}$ Si NMR of 3

$\frac{\substack{\infty \\ 0 \\ 0 \\ 0 \\ 0}}{1}$

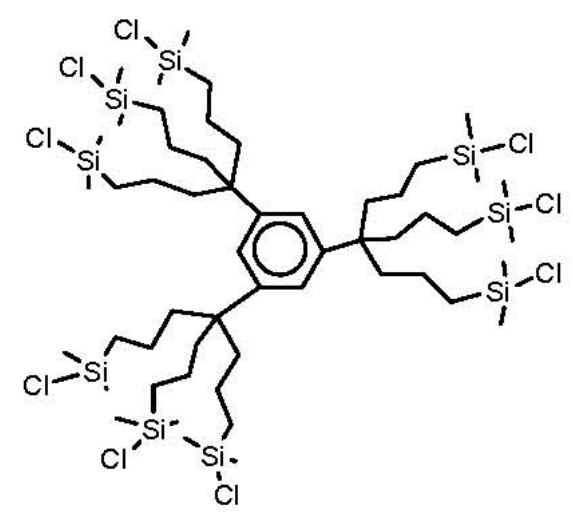

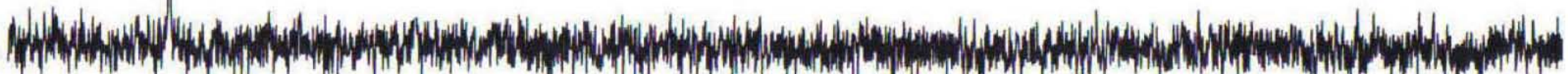

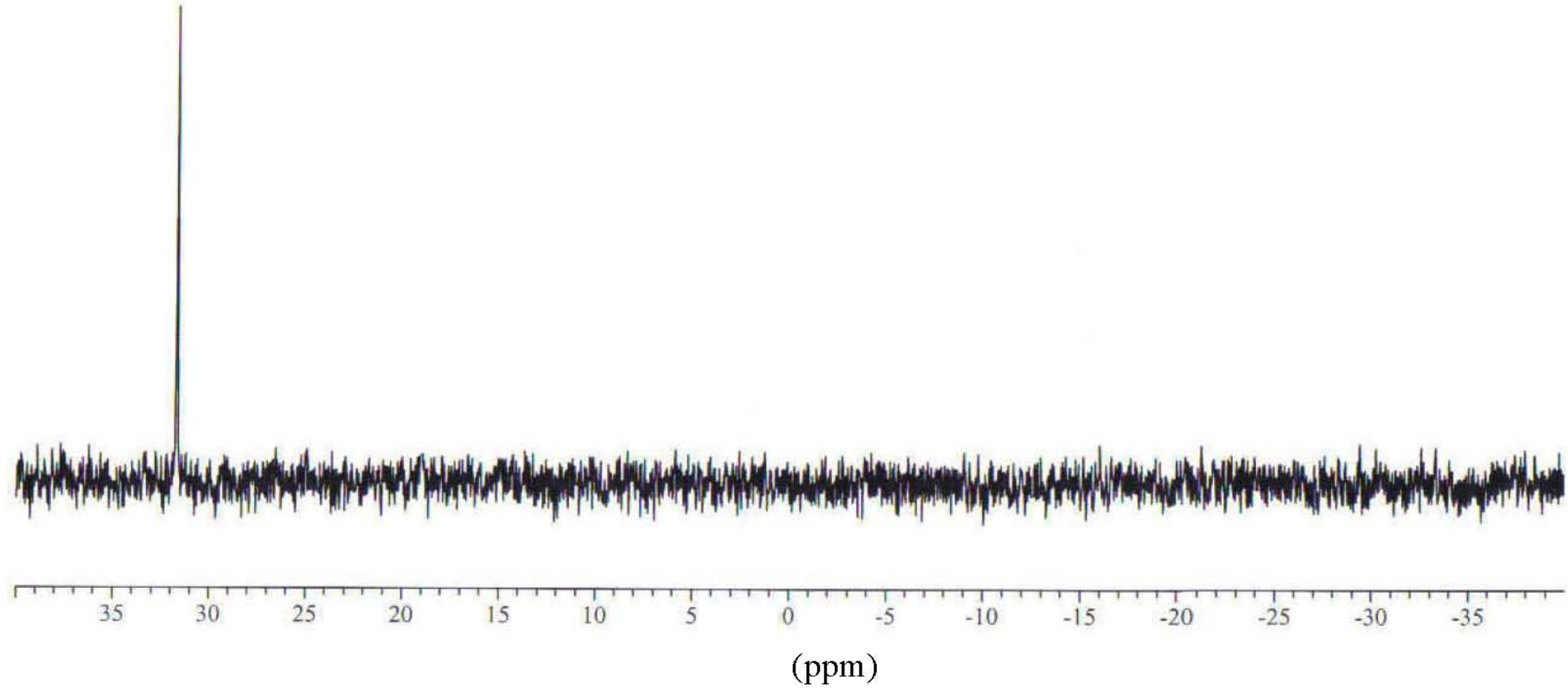




\section{${ }^{29} \mathrm{Si}$ NMR of $\mathbf{5}$}

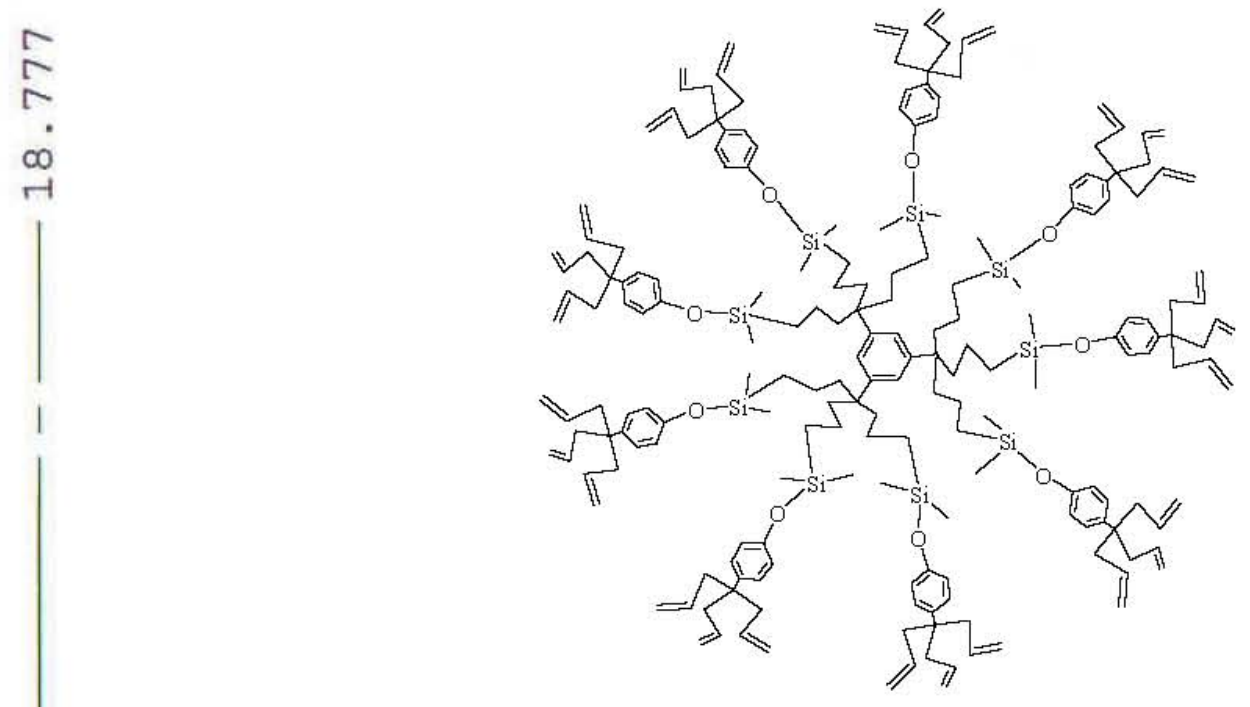

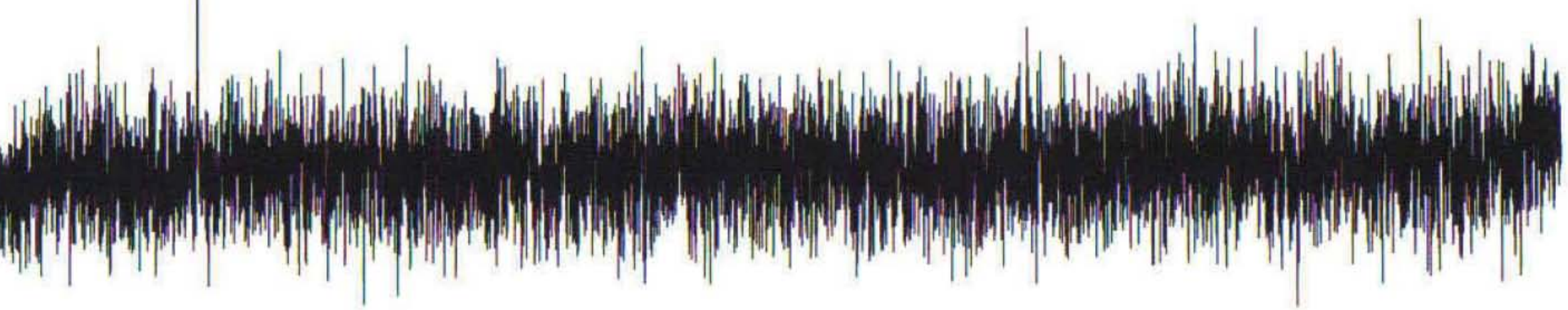

\begin{tabular}{|c|c|c|c|c|c|c|c|}
\hline${ }_{40}^{1}$ & $\begin{array}{c}1 \\
20\end{array}$ & $\begin{array}{l}1 \\
0\end{array}$ & $\begin{array}{l}1 \\
-20\end{array}$ & $\begin{array}{c}1 \\
-40\end{array}$ & $\begin{array}{r}1 \\
-60\end{array}$ & $\begin{array}{c}1 \\
-80\end{array}$ & $\begin{array}{c}1 \\
-100\end{array}$ \\
\hline
\end{tabular}


${ }^{29} \mathrm{Si}$ NMR of 6

$\infty$
0
0

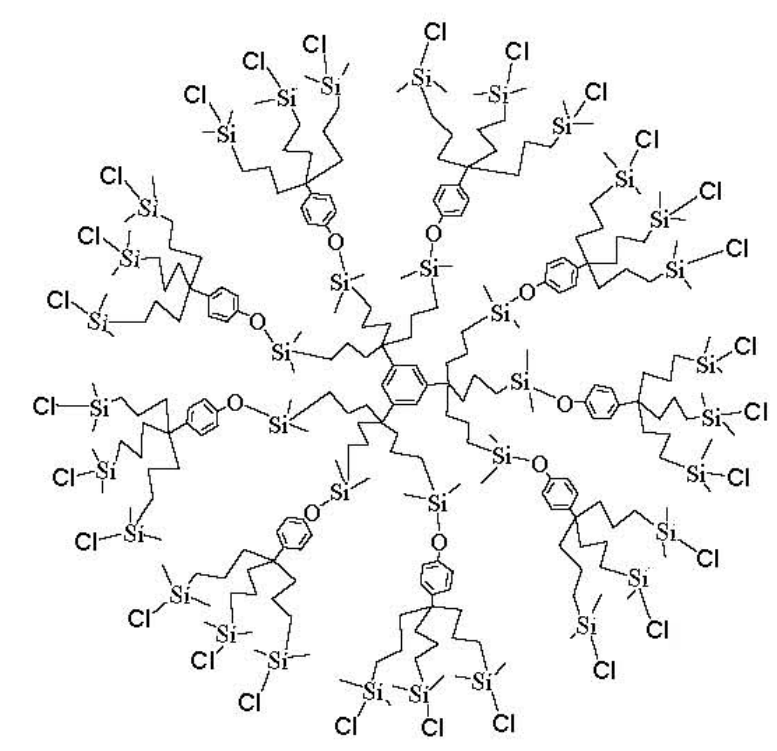




\section{${ }^{29} \mathrm{Si}$ NMR of 7}

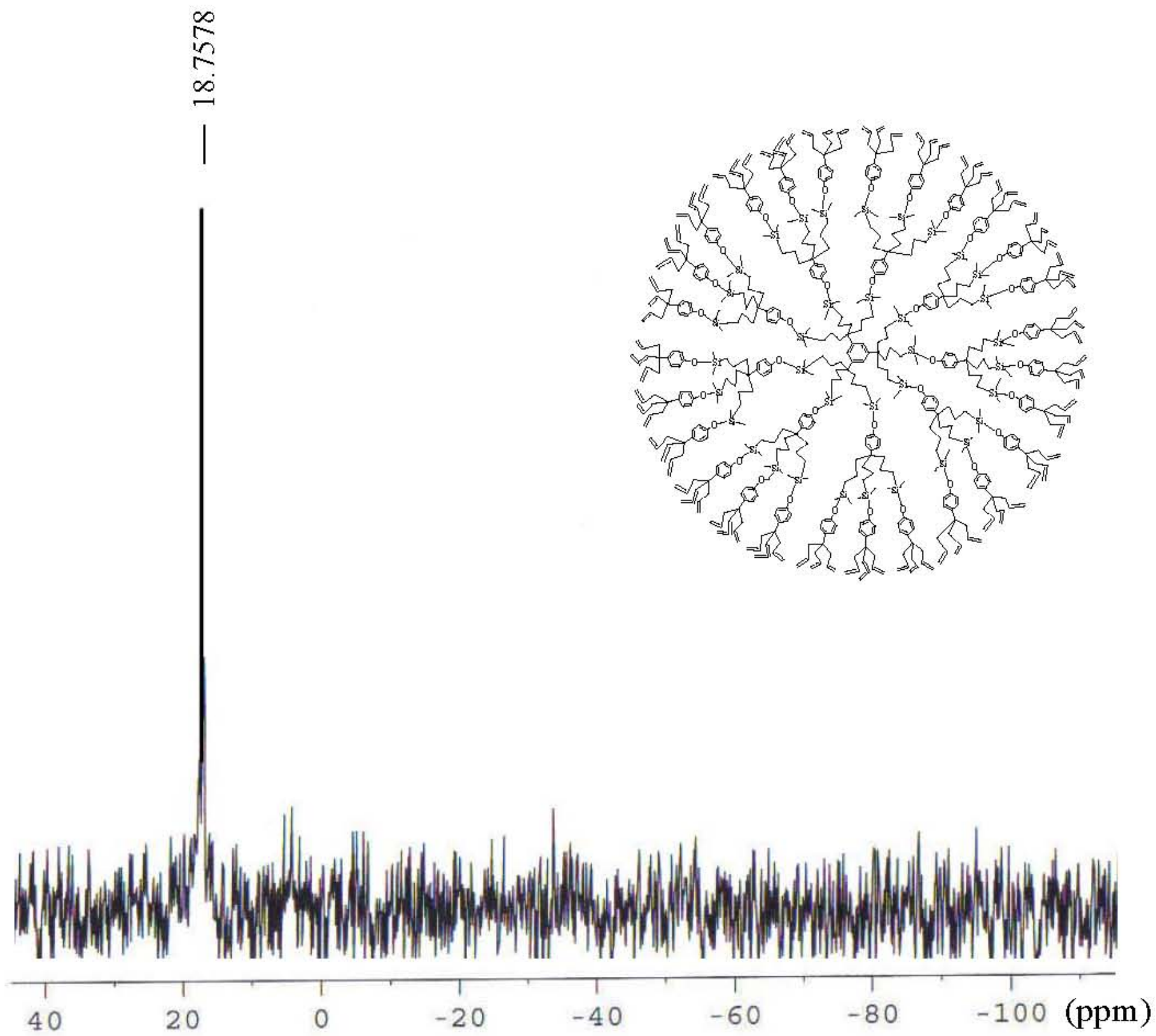




\section{${ }^{29}$ Si NMR of 8}

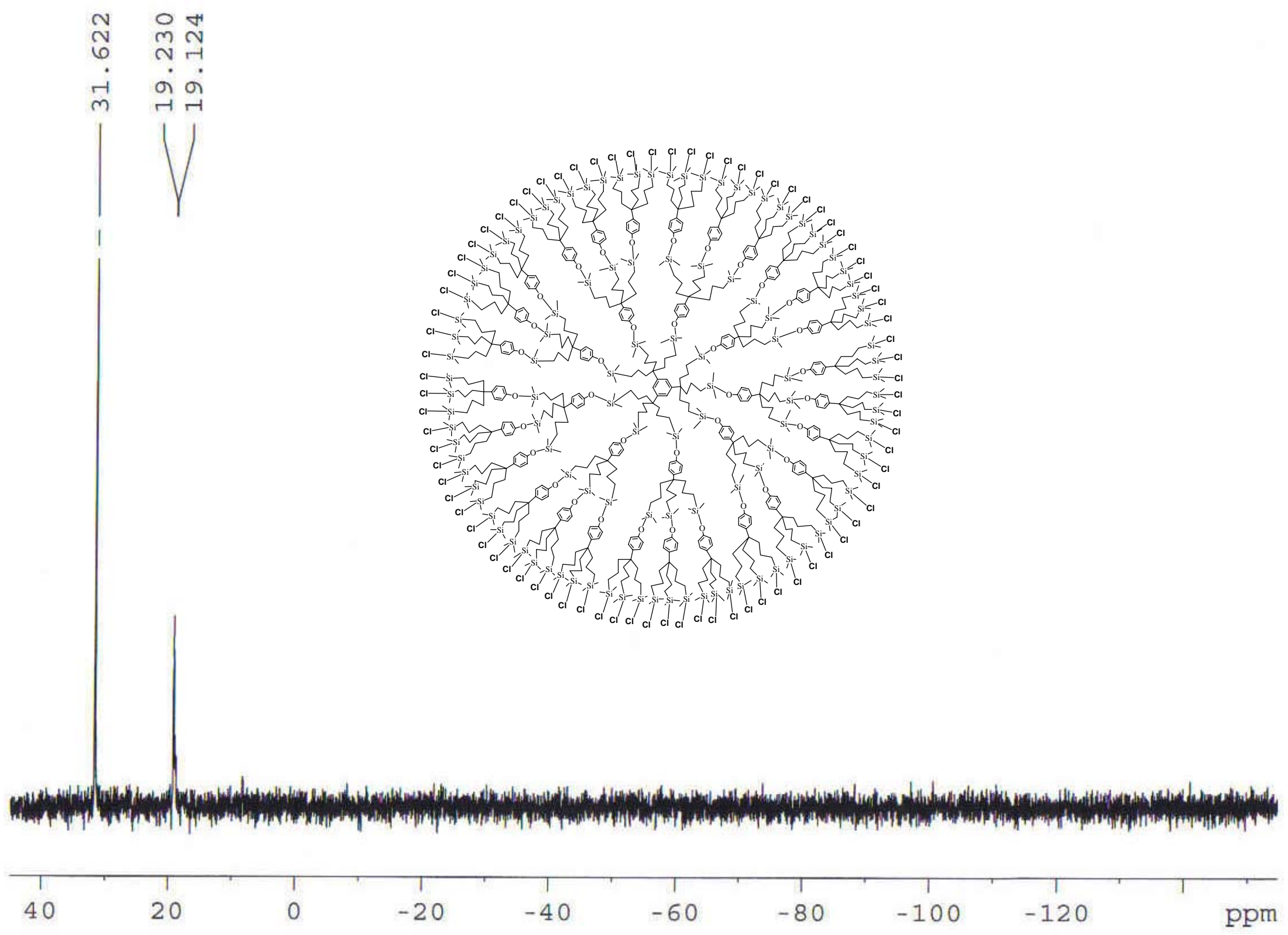


${ }^{29} \mathrm{Si}$ NMR of $\mathbf{9}$

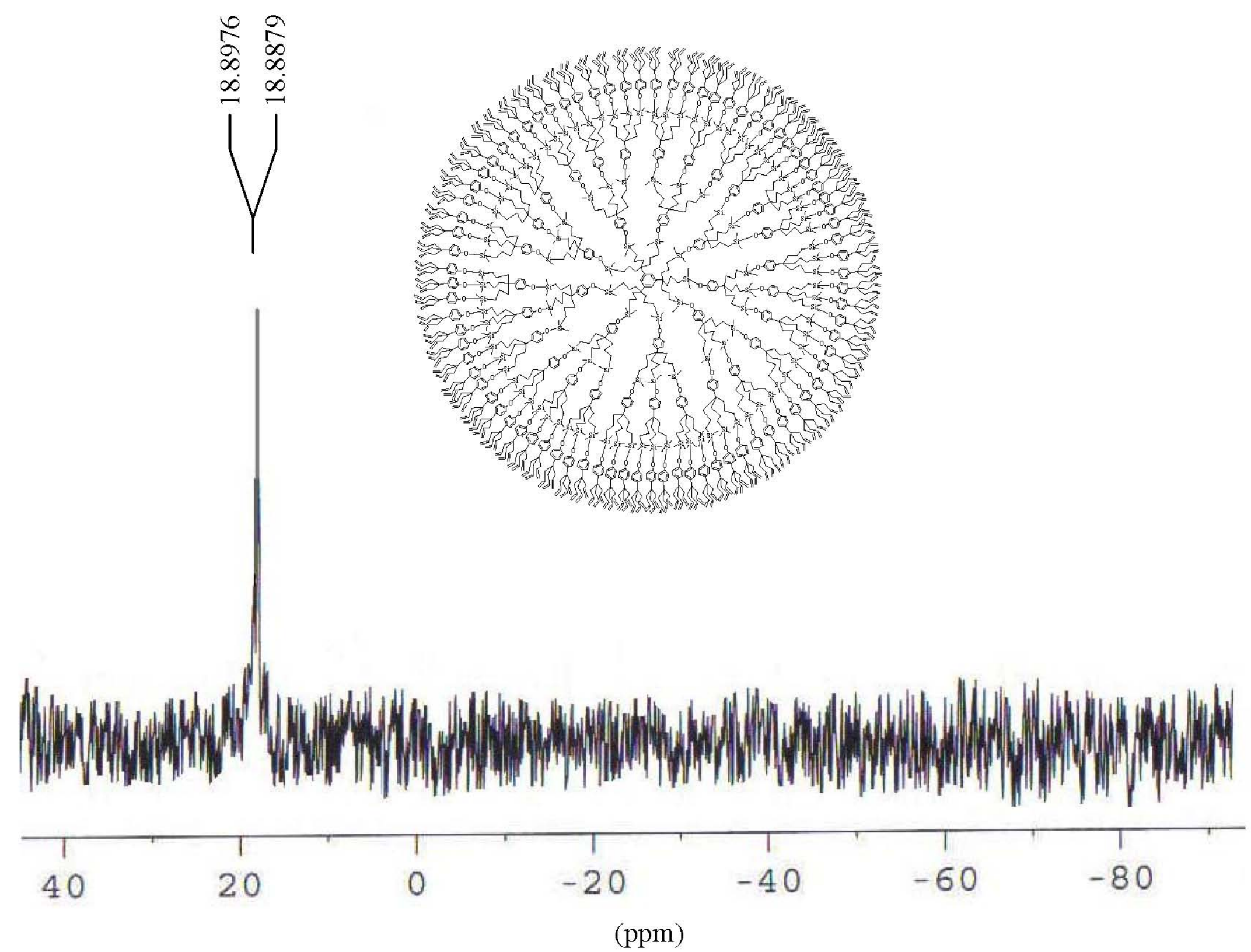




\section{Maldi-Tof Spectra of $\mathbf{5}$}

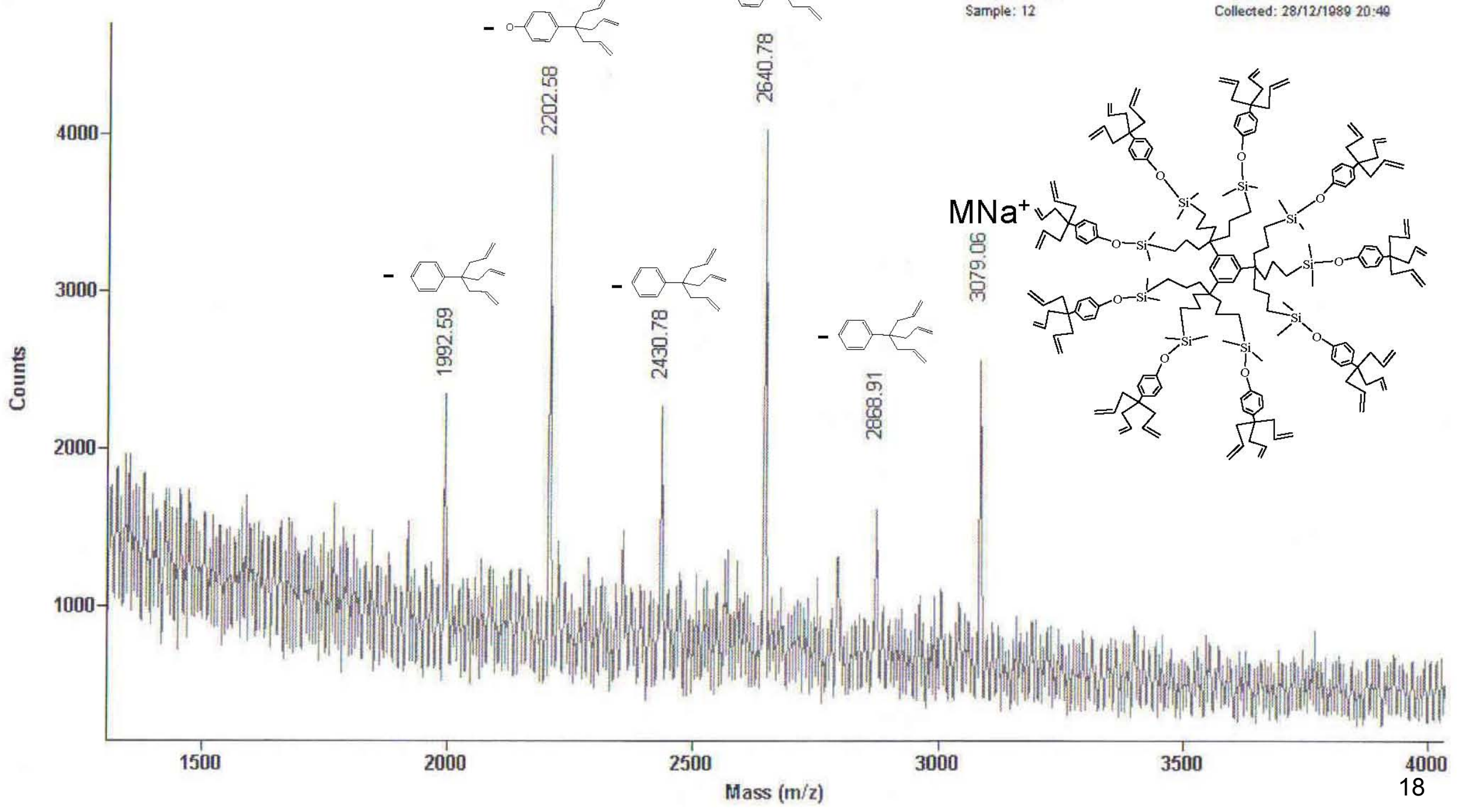

Method: RDEHCCA

Mode: Reflector

Accelerating Whage: 20000

Grid Voltage: 60.000 y

Guide Vurre Voltage: 0.050 \&

Delay: 125 ON

Laser : 2320

Scans Averaged: 256

Pressure: $2.47 \mathrm{e}-07$

Low Mass Gate: 500.0

Timed Ion Selector 2060 O OFF 


\section{Size Exclusion Chromatography}

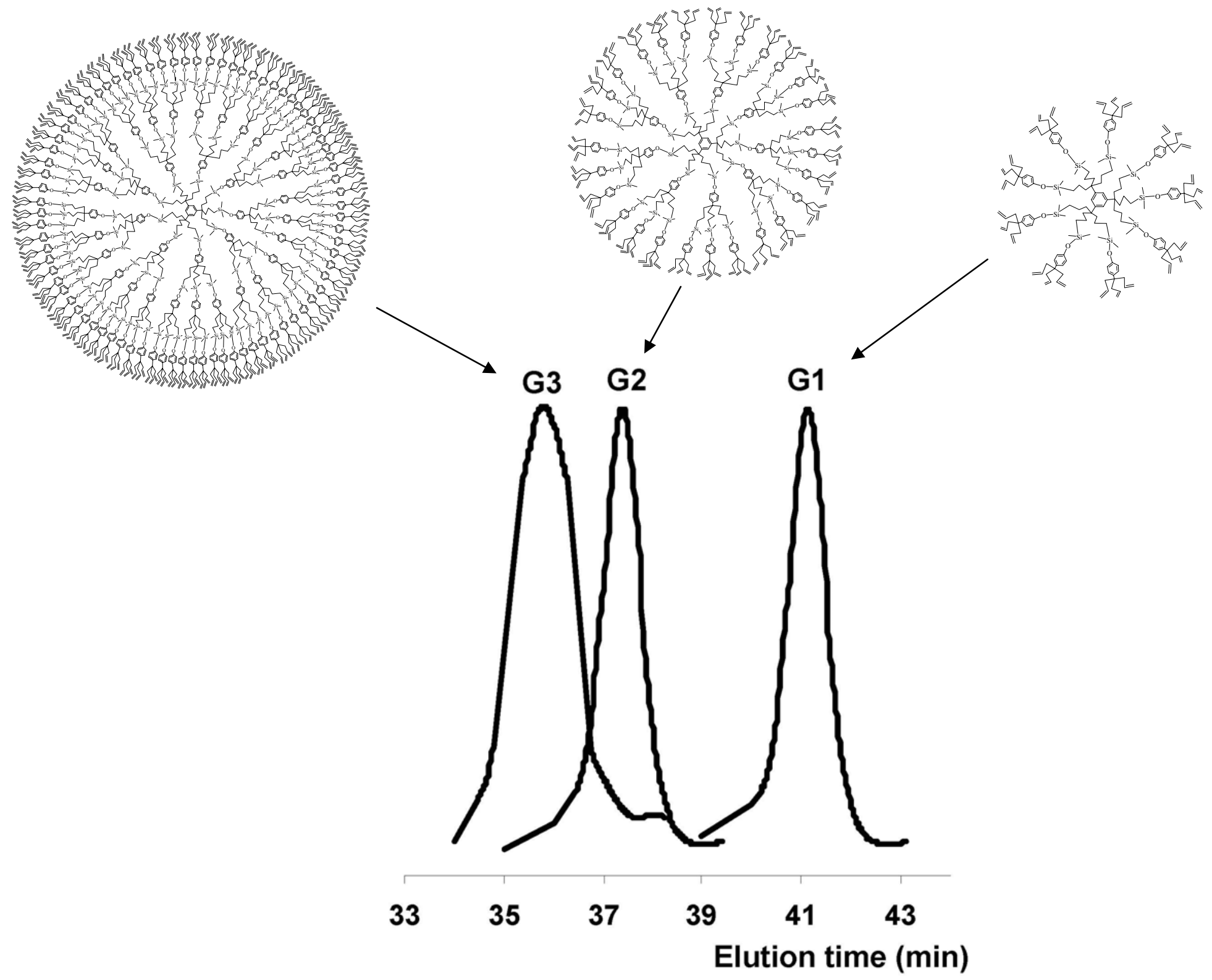

